\title{
Identities in the spirit of Euler
}

\author{
A. Sofo \\ College of Engineering and Science, Victoria University \\ P. O. Box 14428, Melbourne City, Victoria 8001, Australia \\ anthony.sofo@vu.edu.au
}

Received March 26, 2019

Presented by Jesús M.F. Castillo

Accepted September 17, 2019

Abstract: In this paper we develop new identities in the spirit of Euler. We shall investigate and report on new Euler identities of weight $p+2$, for $p$ an odd integer, but with a non unitary argument of the harmonic numbers. Some examples of these Euler identities will be given in terms of Riemann zeta values, Dirichlet values and other special functions.

Key words: Polylogarithm function, recurrence relations, Euler sums, Zeta functions, Dirichlet functions, Multiple zeta values.

AMS Subject Class. (2010): 11M06, 11M32, 33B15.

\section{INTRODUCTION}

In a previous paper, [16] we investigated families of integrals, where the integrand is the product of an inverse trigonometric or inverse hyperbolic trigonometric and the polylogarithmic function,

$$
J(a, \delta, p, m)=\int_{0}^{1} y^{m-1} f(y) \operatorname{Li}_{p}\left(\delta y^{2 a m}\right) \mathrm{d} y,
$$

for $a \in \mathbb{R}^{+}, \delta= \pm 1, p \in \mathbb{N}, m \in \mathbb{R}^{+}$and where $f(y)=\arctan \left(y^{m}\right)$ or $\tanh ^{-1}\left(y^{m}\right)$. It was demonstrated that integrals of products of inverse trigonometric and polylogarithmic functions can be associated with Euler sums. It is well known that integrals with polylogarithmic integrands can be associated with Euler sums. Therefore in the spirit of Euler we shall investigate integrals of the type

$$
I(a, \delta, p, q)=\int_{0}^{1} \frac{\ln x}{x} \operatorname{Li}_{p}(x) \operatorname{Li}_{q}\left(\delta x^{a}\right) \mathrm{d} x
$$

for $a \in \mathbb{R}^{+}, \delta= \pm 1, p \in \mathbb{N}, q \in \mathbb{N}$. Some examples are highlighted, almost none of which are amenable to a computer mathematical package. We shall 
also develop new Euler identities for sums of the type

$$
\sum_{n=1}^{\infty} \frac{H_{\frac{n}{2}}^{(2)}}{n^{p}}, \quad \sum_{n=1}^{\infty} \frac{(-1)^{n+1} H_{\frac{n}{2}}^{(2)}}{n^{p}}, \quad \sum_{n=1}^{\infty} \frac{H_{n-\frac{1}{2}}^{(2)}}{(2 n-1)^{p}}
$$

and again, some examples are highlighted, almost none of which are amenable to a computer mathematical package. This work also extends the results given in [7], where the author examined integrals with positive arguments of the polylogarithm. Devoto and Duke [4] also list many identities of lower order polylogarithmic integrals and their relations to Euler sums. Some other important sources of information on polylogarithm functions are the works of [9], [10], and [16]. The famous Euler identity [5], for unitary argument of the harmonic numbers, states

$$
\begin{aligned}
E U(m)= & \sum_{n=1}^{\infty} \frac{H_{n}}{n^{m}}=\left(\frac{m}{2}+1\right) \zeta(m+1) \\
& -\frac{1}{2} \sum_{j=1}^{m-2} \zeta(m-j) \zeta(j+1) .
\end{aligned}
$$

The famous Euler identity was further extended in the work of [1]. Relatively recently multiple zeta values (MZVs) were studied and developed by [8], [21] and others, for example, [11]. MZVs are defined by

$$
\zeta\left(i_{1}, i_{2}, \ldots, i_{k}\right)=\sum_{n_{1}>n_{2} \cdots>n_{k} \geq 1} \frac{1}{n_{1}^{i_{1}} n_{2}^{i_{2}} \ldots n_{k}^{i_{k}}}
$$

for positive integers $i_{k}$ and $i_{1}>1$ with weight $\sum i_{k}$ and length or depth $k$. For arbitrary $p \in \mathbb{N}$ and $q \geq 2$ the Euler sum

$$
S_{p, q}=\sum_{n=1}^{\infty} \frac{H_{n}^{(p)}}{n^{q}}
$$

is readily expressible in terms of MZVs, that is, $S_{p, q}+S_{q, p}=\zeta(p) \zeta(q)+\zeta(p+q)$. Euler developed many relations, including

$$
\zeta(2,1)=\zeta(3), \quad S_{2,3}=3 \zeta(2) \zeta(3)-\frac{9}{2} \zeta(5)
$$


It appears that at weight eight, $S_{2,6}$ cannot be reduced to zeta values and their products. We also note that we may define alternating MZVs with signs in the numerator as

$$
\zeta\left(\overline{i_{1}}, \overline{i_{2}}, \ldots, \overline{i_{k}}\right)=\sum_{n_{1}>n_{2}>\cdots>n_{k} \geq 1} \frac{(-1)^{n_{1}+n_{2}+\cdots+n_{k}}}{n_{1}^{i_{1}} n_{2}^{i_{2}} \ldots n_{k}^{i_{k}}} .
$$

Therefore an alternating MZV converges unless the first entry is an unbanned one, and we also have $\zeta(\overline{1})=-\ln 2$ and $\zeta(\bar{n})=\eta(n)=\left(1-2^{1-n}\right) \zeta(n)$ for $n \geq 2$, so that, for example

$$
\zeta(\overline{3}, 1, \overline{2})=\sum_{n_{1}>n_{2}>n_{3} \geq 1} \frac{(-1)^{n_{1}+n_{3}}}{n_{1}^{3} n_{2} n_{3}^{2}} .
$$

For arbitrary integer weight $p \geq 1, q \geq 1$ we shall define alternating Euler sums as,

$$
S(p, q):=\sum_{n=1}^{\infty} \frac{(-1)^{n+1} H_{n}^{(p)}}{n^{q}} .
$$

There are some special cases where the linear Euler sum $(1.4)$ is reducible to zeta values. For odd weight $w=(p+q)$ we have,

$$
\begin{aligned}
& B W(p, q)=\sum_{n=1}^{\infty} \frac{H_{n}^{(p)}}{n^{q}}=\frac{1}{2}\left(1+(-1)^{p+1}\right) \zeta(p) \zeta(q) \\
& \quad+(-1)^{p} \sum_{j=1}^{\left[\frac{p}{2}\right]}\left(\begin{array}{c}
p+q-2 j-1 \\
p-1
\end{array}\right) \zeta(p+q-2 j) \zeta(2 j) \\
& \quad+(-1)^{p} \sum_{j=1}^{\left[\frac{p}{2}\right]}\left(\begin{array}{c}
p+q-2 j-1 \\
q-1
\end{array}\right) \zeta(p+q-2 j) \zeta(2 j) \\
& \quad+\frac{\zeta(p+q)}{2}\left(1+(-1)^{p+1}\left(\begin{array}{c}
p+q-1 \\
p
\end{array}\right)+(-1)^{p+1}\left(\begin{array}{c}
p+q-1 \\
q
\end{array}\right)\right)
\end{aligned}
$$

where $[z]$ is the integer part of $z$. For alternating Euler sums and specified odd weights we have some particular identities. Sitaramachandra Rao, [12 gave the identity, for $S(p, q)$, when $p=1$ and for odd weight $1+q$ as,

$$
2 S(1, q)=(1+q) \eta(1+q)-\zeta(1+q)-2 \sum_{j=1}^{\frac{q}{2}-1} \eta(2 j) \zeta(1+q-2 j)
$$


and in another special case, gave the integral

$$
S(1,1+2 q)=\frac{1}{(2 q) !} \int_{0}^{1} \frac{\ln ^{2 q}(x) \ln (1+x)}{x(1+x)} \mathrm{d} x .
$$

In the case where $p$ and $q$ are both positive integers and $p+q$ is an odd integer, Flajolet and Salvy [6] gave the identity:

$$
\begin{aligned}
2 S(p, q)=(1 & \left.-(-1)^{p}\right) \zeta(p) \eta(q) \\
& +2(-1)^{p} \sum_{i+2 k=q}\left(\begin{array}{c}
p+i-1 \\
p-1
\end{array}\right) \zeta(p+i) \eta(2 k) \\
& +\eta(p+q)-2 \sum_{j+2 k=p}\left(\begin{array}{c}
q+j-1 \\
q-1
\end{array}\right)(-1)^{j} \eta(q+j) \eta(2 k),
\end{aligned}
$$

where $\eta(0)=\frac{1}{2}, \eta(1)=\ln 2, \zeta(1)=0$, and $\zeta(0)=-\frac{1}{2}$ in accordance with the analytic continuation of the Riemann zeta function. We define the alternating zeta function (or Dirichlet eta function) $\eta(z)$ as

$$
\eta(z):=\sum_{n=1}^{\infty} \frac{(-1)^{n+1}}{n^{z}}=\left(1-2^{1-z}\right) \zeta(z) .
$$

The following Euler identities for harmonic numbers at half integer values have been given in [19].

Lemma 1. For $\delta= \pm 1, a \in \mathbb{R}^{+}, r \in \mathbb{N}$ and $m$ a positive odd integer,

$$
W(a, \delta, m, r)=\sum_{n \geq 1} \frac{\delta^{n+1} H_{a n}^{(r)}}{n^{m}}
$$

then

$$
\begin{aligned}
W\left(\frac{1}{2}, 1, m, 1\right)= & E U(m)+(-1)^{m+1} S(1, m) \\
& +\sum_{k=2}^{m-1}(-1)^{m-k} \zeta(k) \eta(m+1-k),
\end{aligned}
$$

and

$$
\begin{aligned}
W\left(\frac{1}{2},-1, m, 1\right)= & \left(1-2^{1-m}\right) E U(m)+(-1)^{m+1} S(1, m) \\
& +\sum_{k=2}^{m-1}(-1)^{m-k} \zeta(k) \eta(m+1-k) .
\end{aligned}
$$


Therefore the main aim of this paper is to develop new Euler identities for the sums (1.2) and represent the solution of the integral (1.1), in terms of special functions, for various values of the parameters $(a, \delta, p, q)$. First we define some special functions that we will encounter in the body of this paper. The Lerch transcendent,

$$
\Phi(z, t, a)=\sum_{m=0}^{\infty} \frac{z^{m}}{(m+a)^{t}}
$$

is defined for $|z|<1$ and $\Re(a)>0$ and satisfies the recurrence

$$
\Phi(z, t, a)=z \Phi(z, t, a+1)+a^{-t} .
$$

The Lerch transcendent generalizes the Hurwitz zeta function at $z=1$,

$$
\Phi(1, t, a)=\sum_{m=0}^{\infty} \frac{1}{(m+a)^{t}}
$$

and the polylogarithm, or de-Jonquière's function, when $a=1$,

$$
\operatorname{Li}_{t}(z):=\sum_{m=1}^{\infty} \frac{z^{m}}{m^{t}}, \quad t \in \mathbb{C} \text { when }|z|<1 ; \quad \Re(t)>1 \text { when }|z|=1 .
$$

Let

$$
H_{n}=\sum_{r=1}^{n} \frac{1}{r}=\int_{0}^{1} \frac{1-t^{n}}{1-t} \mathrm{~d} t=\gamma+\psi(n+1)=\sum_{j=1}^{\infty} \frac{n}{j(j+n)}, \quad H_{0}:=0
$$

be the $n t h$ harmonic number, where $\gamma$ denotes the Euler-Mascheroni constant, $H_{n}^{(m)}=\sum_{r=1}^{n} \frac{1}{r^{m}}$ is the $m^{\text {th }}$ order harmonic number and $\psi(z)$ is the digamma (or psi) function defined by

$$
\psi(z):=\frac{\mathrm{d}}{\mathrm{d} z}\{\log \Gamma(z)\}=\frac{\Gamma^{\prime}(z)}{\Gamma(z)} \quad \text { and } \quad \psi(1+z)=\psi(z)+\frac{1}{z},
$$

moreover,

$$
\psi(z)=-\gamma+\sum_{n=0}^{\infty}\left(\frac{1}{n+1}-\frac{1}{n+z}\right) .
$$

The polygamma function

$$
\psi^{(k)}(z)=\frac{\mathrm{d}^{k}}{\mathrm{~d} z^{k}}\{\psi(z)\}=(-1)^{k+1} k ! \sum_{r=0}^{\infty} \frac{1}{(r+z)^{k+1}}
$$


and has the recurrence

$$
\psi^{(k)}(z+1)=\psi^{(k)}(z)+\frac{(-1)^{k} k !}{z^{k+1}} .
$$

The connection of the polygamma function with harmonic numbers is,

$$
H_{z}^{(\alpha+1)}=\zeta(\alpha+1)+\frac{(-1)^{\alpha}}{\alpha !} \psi^{(\alpha)}(z+1), \quad z \neq\{-1,-2,-3, \ldots\},
$$

and the multiplication formula is

$$
\psi^{(k)}(p z)=\delta_{m, 0} \ln p+\frac{1}{p^{k+1}} \sum_{j=0}^{p-1} \psi^{(k)}\left(z+\frac{j}{p}\right)
$$

for $p$ a positive integer and $\delta_{p, k}$ is the Kronecker delta. The work in this paper also extends the results of [7], 20]. Other works including, [2], 3], [13], [14], [15], [17, and [18] cite many identities of polylogarithmic integrals and Euler sums.

\section{INTEGRAL IDENTITIES AND EULER SUMS}

Theorem 1. For $a \in \mathbb{R}^{+}, \delta=\{-1,1\}, p, q$, positive integers, then

$$
\begin{aligned}
I(a, \delta, p, q)= & \int_{0}^{1} \frac{\ln x \operatorname{Li}_{p}(x) \operatorname{Li}_{q}\left(\delta x^{a}\right)}{x} \mathrm{~d} x \\
= & \frac{(-1)^{p+1}}{a^{p}} \zeta(2) \operatorname{Li}_{p+q}(\delta) \\
& +\frac{(-1)^{p} p}{a^{p+1}} \sum_{n=1}^{\infty} \frac{\delta^{n} H_{a n}}{n^{p+q+1}}+\frac{(-1)^{p}}{a^{p}} \sum_{n=1}^{\infty} \frac{\delta^{n} H_{a n}^{(2)}}{n^{p+q}} \\
& -\sum_{k=2}^{p} \frac{(-1)^{p-k}(p+1-k)}{a^{p+2-k}} \zeta(k) \operatorname{Li}_{p+q+2-k}(\delta) .
\end{aligned}
$$

where $L i_{p+q}(\delta)$ is the polylogarithm, $H_{a n}$ and $H_{a n}^{(2)}$ are the harmonic numbers.

Proof. By the definition of the polylogarithmic function we have

$$
\begin{aligned}
I(a, \delta, p, q) & =\sum_{n=1}^{\infty} \frac{\delta^{n}}{n^{q}} \sum_{j=1}^{\infty} \frac{1}{j^{p}} \int_{0}^{1} x^{j+a n-1} \ln x \mathrm{~d} x \\
& =-\sum_{n=1}^{\infty} \frac{\delta^{n}}{n^{q}} \sum_{j=1}^{\infty} \frac{1}{j^{p}(j+a n)^{2}}
\end{aligned}
$$


and by partial fraction decomposition,

$$
I(a, \delta, p, q)=-\sum_{n=1}^{\infty} \frac{\delta^{n}}{n^{q}} \sum_{j=1}^{\infty}\left(\begin{array}{c}
\frac{p(-1)^{p+1}}{(a n)^{p} j(j+a n)}+\frac{(-1)^{p}}{(a n)^{p}(j+a n)^{2}} \\
+\sum_{k=2}^{p} \frac{(-1)^{p-k}(p+1-k)}{(a n)^{p+2-k} j^{k}}
\end{array}\right) .
$$

Now

$$
\begin{aligned}
I(a, \delta, p, q)= & -\sum_{n=1}^{\infty} \frac{\delta^{n}}{n^{q}}\left(\begin{array}{c}
\frac{p(-1)^{p+1} H_{a n}}{(a n)^{p+1}}+\frac{(-1)^{p} \psi^{\prime}(a n+1)}{(a n)^{p}} \\
+\sum_{k=2}^{p} \frac{(-1)^{p-k}(p+1-k)}{(a n)^{p+2-k}} \zeta(k)
\end{array}\right) \\
= & \frac{(-1)^{p+1}}{a^{p}} \zeta(2) \operatorname{Li}_{p+q}(\delta)+\frac{(-1)^{p} p}{a^{p+1}} \sum_{n=1}^{\infty} \frac{\delta^{n} H_{a n}}{n^{p+q+1}} \\
& +\frac{(-1)^{p}}{a^{p}} \sum_{n=1}^{\infty} \frac{\delta^{n} H_{a n}^{(2)}}{n^{p+q}} \\
& -\sum_{k=2}^{p} \frac{(-1)^{p-k}(p+1-k)}{a^{p+2-k}} \zeta(k) \operatorname{Li}_{p+q+2-k}(\delta),
\end{aligned}
$$

and Theorem 1 is proved.

In the next few corollaries we investigate various special values of the parameters $(a, \delta, p, q)$ which will yield solutions to $I(a, \delta, p, q)$ that are expressible in terms of the Riemann zeta and other special functions. We shall also present new Euler type identities for the sums (1.2).

Corollary 1. For $a=1, \delta=1, p, q$, positive integers with arbitrary weight $p+q$, then

$$
\begin{aligned}
I(1,1, p, q)= & \int_{0}^{1} \frac{\ln x \operatorname{Li}_{p}(x) \operatorname{Li}_{q}(x)}{x} \mathrm{~d} x \\
= & (-1)^{p}\left(S_{2, p+q}+p S_{1, p+q+1}\right)+(-1)^{p+1} \zeta(2) \zeta(p+q) \\
& -\sum_{k=2}^{p}(-1)^{p-k}(p+1-k) \zeta(k) \zeta(p+q+2-k),
\end{aligned}
$$

where $S_{a, b}$ is the linear Euler sum (1.4). 
Proof. Here we note that $\operatorname{Li}_{m}(1)=\zeta(m)$ and the sums $S_{2, p+q}=\sum_{n=1}^{\infty} \frac{H_{n}^{(2)}}{n^{p+q}}$ and $S_{1, p+q+1}=\sum_{n=1}^{\infty} \frac{H_{n}}{n^{p+q+1}}$.

Remark 1. For $a=1, \delta=1, p, q$, positive integers with $p+q$ an odd integer, then

$$
\begin{aligned}
I(1,1, p, q)= & \int_{0}^{1} \frac{\ln x \operatorname{Li}_{p}(x) \operatorname{Li}_{q}(x)}{x} \mathrm{~d} x \\
= & p(-1)^{p} E U(p+q+1)+(-1)^{p} B W(2, p+q) \\
& +(-1)^{p+1} \zeta(2) \zeta(p+q) \\
& -\sum_{k=2}^{p}(-1)^{p-k}(p+1-k) \zeta(k) \zeta(p+q+2-k),
\end{aligned}
$$

where $E U(\cdot)$ is the Euler identity (1.3) and $B W(\cdot, \cdot)$ is the identity (1.6).

EXAMPLE 1.

$$
\begin{aligned}
& I(1,1,4,2)=I(1,1,2,4)=S_{2,6}-2 \zeta(3) \zeta(5)+\frac{7}{6} \zeta(8), \\
& I(1,1,4,4)=S_{2,8}-2 \zeta(3) \zeta(7)+\frac{33}{10} \zeta(10)-2 \zeta^{2}(5), \\
& I(1,1,4,5)=I(1,1,5,4)=\zeta(4) \zeta(7)+\zeta(2) \zeta(9)-3 \zeta(11) .
\end{aligned}
$$

Corollary 2. For $a=1, \delta=-1, p, q$, positive integers, then

$$
\begin{aligned}
I(1,-1, p, q)= & \int_{0}^{1} \frac{\ln x \operatorname{Li}_{p}(x) \operatorname{Li}_{q}(-x)}{x} \mathrm{~d} x \\
= & p(-1)^{p+1} S(1, p+q+1) \\
& +(-1)^{p+1} S(2, p+q)+(-1)^{p} \zeta(2) \eta(p+q) \\
& +\sum_{k=2}^{p}(-1)^{p-k}(p+1-k) \zeta(k) \eta(p+q+2-k),
\end{aligned}
$$

where $\eta(\cdot)$ is the Dirichlet Eta function and $S(\cdot, \cdot)$ is the alternating linear Euler sum. We note that when we have odd weight $(p+q), S(\cdot, \cdot)$ may be replaced with the identity 1.8 . 
Proof. Here we note that $\operatorname{Li}_{m}(-1)=-\eta(m)$ and the sum $S(m, p+q)=$ $\sum_{n=1}^{\infty} \frac{(-1)^{n+1} H_{n}^{(m)}}{n^{p+q}}$ and may be replaced with the identity 1.8$)$ in the case when we have odd weight $(p+q)$.

EXAMPLE 2.

$$
\begin{aligned}
I(1,-1,2,4) & =2 \zeta(2) \eta(6)-S(2,6)-2 S(1,7), \\
I(1,-1,4,2) & =-\frac{359}{48} \zeta(8)-2 \zeta(3) \eta(5)-S(2,6)-4 S(1,7), \\
I(1,-1,2,3) & =\frac{43}{32} \zeta(2) \zeta(5)-2 \zeta(7), \\
I(1,-1,3,4) & =\frac{7}{8} \zeta(4) \zeta(5)+\frac{5}{2} \zeta(9)-\frac{249}{128} \zeta(2) \zeta(7) .
\end{aligned}
$$

Corollary 3. For $a=2, \delta=1, p, q$, positive integers, then

$$
\begin{aligned}
I(2,1, p, q)= & \int_{0}^{1} \frac{\ln x \operatorname{Li}_{p}(x) \operatorname{Li}_{q}\left(x^{2}\right)}{x} \mathrm{~d} x \\
= & \frac{(-1)^{p+1}}{2^{p}} \zeta(2) \zeta(p+q) \\
& +p(-1)^{p} 2^{q-1}\left(S_{1, p+q+1}-S(1, p+q+1)\right) \\
& +(-1)^{p} 2^{q-1}\left(S_{2, p+q}-S(2, p+q)\right) \\
& -(-1)^{p} \sum_{k=2}^{p} \frac{(-1)^{k}(p+1-k)}{2^{p+2-k}} \zeta(k) \zeta(p+q+2-k),
\end{aligned}
$$

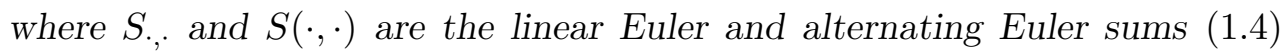
and (1.5) respectively. In the case when we have odd weight $(p+q)$ then we may utilize $S_{1, p+q+1}=E U(p+q+1)$ is the Euler identity (1.3), $S_{2, p+q}=$ $B W(2, p+q)$ is the identity (1.6) and $S(\cdot, \cdot)$ is obtained from the identity (1.8).

Proof. Here we note that $\operatorname{Li}_{m}(1)=\zeta(m)$ and the sums $S_{\text {., }}$ and $S(\cdot, \cdot)$ are the linear Euler and alternating Euler sums (1.4) and (1.5) respectively. In the case of odd weight $(p+q)$ the sums $S_{1, p+q+1}=\sum_{n=1}^{\infty} \frac{H_{n}}{n^{p+q+1}}, S_{2, p+q}=$ $B W(2, p+q)=\sum_{n=1}^{\infty} \frac{H_{n}^{(2)}}{n^{p+q}}$ and $S(a, b)=\sum_{n=1}^{\infty} \frac{(-1)^{n+1} H_{n}^{(a)}}{n^{b}}$. 
EXAMPLE 3 .

$$
\begin{aligned}
I(2,1,3,3) & =\frac{47}{4} \zeta(3) \zeta(5)-\frac{211}{8} \zeta(8)-4 S_{2,6}+4 S(2,6)+12 S(1,7), \\
I(2,1,4,2) & =\frac{415}{24} \zeta(8)-\frac{31}{4} \zeta(3) \zeta(5)+2 S_{2,6}-2 S(2,6)-8 S(1,7), \\
I(2,1,3,2) & =\frac{11}{32} \zeta(5)-\frac{7}{16} \zeta(2) \zeta(3), \\
I(2,1,0, q) & =2^{q-1}(E U(q)-S(1, q)), \\
I(2,1,5,0) & =\frac{107}{256} \zeta(7)+\frac{1}{4} \zeta(4) \zeta(3)-\frac{37}{64} \zeta(5) \zeta(2) .
\end{aligned}
$$

The aim now, is to obtain the new Euler identities for the sums (1.2), hence consider the following corollary.

Corollary 4. From Corollary 3, let $q=0, \delta= \pm 1$, and $p$, a positive odd integer, then

$$
\begin{aligned}
W\left(\frac{1}{2}, 1, p, 2\right)= & \sum_{n \geq 1} \frac{H_{\frac{n}{2}}^{(2)}}{n^{p}} \\
= & p(-1)^{p+1} 2^{2-p} \zeta(2) \zeta(p)+2 p(-1)^{p}(E U(p+1)-S(1, p+1)) \\
& +2(-1)^{p}(B W(2, p)-S(2, p)) \\
& +(-1)^{p+1} \sum_{k=3}^{p-1} \frac{(-1)^{k}(p+1-k)}{2^{p-k}} \zeta(k) \zeta(p+2-k)
\end{aligned}
$$

where $E U(\cdot), S(\cdot, \cdot)$ and $B W(\cdot, \cdot)$ are the same as in Corollary 3 .

Proof. Proceeding as in Theorem 1,

$$
\begin{aligned}
I(a, \delta, p, 0) & =\int_{0}^{1} \frac{\ln x \operatorname{Li}_{p}(x) \operatorname{Li}_{0}\left(\delta x^{a}\right)}{x} \mathrm{~d} x \\
& =-\frac{\delta}{a^{2}} \sum_{n \geq 1} \frac{1}{n^{p}} \sum_{j \geq 1} \frac{\delta^{j}}{(n+a j)^{2}}=-\frac{\delta}{a^{2}} \sum_{n \geq 1} \frac{1}{n^{p}} \Phi\left(\delta, 2,1+\frac{n}{a}\right),
\end{aligned}
$$

where $\Phi\left(\delta, 2,1+\frac{n}{a}\right)$ is the Lerch transcendent. We have,

$$
I(a, \delta, p, 0)= \begin{cases}-\frac{1}{a^{2}} \sum_{n \geq 1} \frac{\psi^{\prime}\left(\frac{n}{a}+1\right)}{n^{p}} & \text { for } \delta=1, \\ \frac{1}{4 a^{2}} \sum_{n \geq 1} \frac{\psi^{\prime}\left(\frac{n}{a}+1\right)}{n^{p}}\left(\psi^{\prime}\left(\frac{a+n}{2 a}\right)-\psi^{\prime}\left(\frac{2 q+n}{2 a}\right)\right) & \text { for } \delta=-1 .\end{cases}
$$


Now for $a=2$ and $\delta=1$, we have

$$
I(2,1, p, 0)=-\frac{1}{4} \sum_{n \geq 1} \frac{\psi^{\prime}\left(\frac{n}{2}+1\right)}{n^{p}}=\frac{1}{4} \sum_{n \geq 1} \frac{H_{\frac{n}{2}}^{(2)}}{n^{p}}-\frac{1}{4} \zeta(2) \zeta(p)
$$

and equating with (2.5) we obtain the desired result (2.6). Also, since

$$
\sum_{n \geq 1} \frac{H_{n}^{(2)}}{n^{p}}=2^{1-p} \sum_{n \geq 1} \frac{H_{\frac{n}{2}}^{(2)}}{n^{p}}\left(1-(-1)^{n+1}\right)
$$

we obtain the second Euler identity

$$
\begin{aligned}
W\left(\frac{1}{2},-1, p, 2\right)= & \sum_{n \geq 1} \frac{(-1)^{n+1} H_{\frac{n}{2}}^{(2)}}{n^{p}} \\
= & W\left(\frac{1}{2}, 1, p, 2\right)-2^{1-p} B W(2, p) \\
= & \left(1+(-2)^{p+1}\right) \zeta(2) \zeta(p)-2 p W\left(\frac{1}{2},-1, p+1,1\right) \\
& +(-2)^{2-p} W(2,1, p, 2)+(-2)^{1-p} B W(2, p) \\
& +\sum_{k=2}^{p}(-2)^{2-k}(p+1-k) \zeta(k) \eta(p+2-k) .
\end{aligned}
$$

Similarly, for the third Euler sum identity in 1.2 we have

$$
\sum_{n=1}^{\infty} \frac{H_{n-\frac{1}{2}}^{(2)}}{(2 n-1)^{p}}=\frac{1}{2}\left(W\left(\frac{1}{2}, 1, p, 2\right)+W\left(\frac{1}{2},-1, p, 2\right)\right) .
$$

EXAMPLE 4.

$$
\begin{aligned}
\sum_{n \geq 1} \frac{H_{\frac{n}{2}}}{n^{6}} & =\frac{135}{128} \zeta(7)-\frac{1}{16} \zeta(2) \zeta(5)-\frac{1}{4} \zeta(3) \zeta(4), \\
\sum_{n \geq 1} \frac{(-1)^{n+1} H_{\frac{n}{2}}}{n^{6}} & =\frac{119}{128} \zeta(7)-\frac{1}{32} \zeta(2) \zeta(5)-\frac{7}{32} \zeta(3) \zeta(4),
\end{aligned}
$$




$$
\begin{aligned}
\sum_{n \geq 1} \frac{H_{\frac{n}{2}}^{(2)}}{n^{5}} & =\zeta(3) \zeta(4)-\frac{21}{16} \zeta(2) \zeta(5)+\frac{107}{64} \zeta(7), \\
\sum_{n \geq 1} \frac{(-1)^{n+1} H_{\frac{n}{2}}^{(2)}}{n^{5}} & =\frac{7}{8} \zeta(3) \zeta(4)-\frac{13}{8} \zeta(2) \zeta(5)+\frac{147}{64} \zeta(7), \\
\sum_{n=1}^{\infty} \frac{H_{n-\frac{1}{2}}^{(2)}}{(2 n-1)^{5}} & =\frac{15}{16} \zeta(3) \zeta(4)-\frac{47}{16} \zeta(2) \zeta(5)+\frac{127}{64} \zeta(7) .
\end{aligned}
$$

Corollary 5. For $a=\frac{1}{2}, \delta= \pm 1, p, q$, positive integers with $p+q$ an odd integer, then

$$
\begin{aligned}
& I\left(\frac{1}{2}, \delta, p, q\right)=\int_{0}^{1} \frac{\ln x \operatorname{Li}_{p}(x) \operatorname{Li}_{q}\left(\delta x^{\frac{1}{2}}\right)}{x} \mathrm{~d} x \\
& \quad=\left\{\begin{array}{l}
2 p(-2)^{p} W\left(\frac{1}{2}, 1, p+q+1,1\right)-(-2)^{p} \zeta(2) \zeta(p+q) \\
+(-2)^{p} W\left(\frac{1}{2}, 1, p+q, 2\right) \\
-\sum_{k=2}^{p}(-1)^{p-k}(p+1-k) \zeta(k) \zeta(p+q+2-k) \\
(-2)^{p+1} W\left(\frac{1}{2},-1, p+q+1,1\right)+(-2)^{p} \zeta(2) \eta(p+q) \\
+(-2)^{p} W\left(\frac{1}{2},-1, p+q, 2\right) \\
+\sum_{k=2}^{p}(-1)^{p-k}(p+1-k) \zeta(k) \eta(p+q+2-k)
\end{array}\right. \\
&
\end{aligned}
$$

where $W(\cdot, \cdot, \cdot, \cdot)$ is evaluated from Corollary (4).

Proof. The proof follows from 2.1.

EXAMPLE 5. In these examples we utilize some results from Example 4:

$$
I\left(\frac{1}{2}, 1,5,0\right)=16 \zeta(3) \zeta(4)+218 \zeta(2) \zeta(5)-391 \zeta(7),
$$




$$
\begin{aligned}
I\left(\frac{1}{2},-1,5,0\right) & =371 \zeta(7)-12 \zeta(3) \zeta(4)-210 \zeta(2) \zeta(5), \\
I\left(\frac{1}{2}, 1,0,5\right) & =\frac{107}{16} \zeta(7)+\zeta(3) \zeta(4)-\frac{37}{16} \zeta(2) \zeta(5), \\
I\left(\frac{1}{2},-1,0,5\right) & =-\frac{147}{16} \zeta(7)-\frac{7}{8} \zeta(3) \zeta(4)+\frac{41}{16} \zeta(2) \zeta(5), \\
I\left(\frac{1}{2}, 1,3,2\right) & =\frac{75}{2} \zeta(2) \zeta(5)-64 \zeta(7), \\
I\left(\frac{1}{2},-1,3,2\right) & =63 \zeta(7)-37 \zeta(2) \zeta(5) .
\end{aligned}
$$

Remark 2. The integral $I(a, \delta, p, q)$ has been represented in terms of special functions. For particular values of the constants $(a, \delta, p, q)$ the integral (1.1) has been expressed in closed form in terms of Riemann zeta and Dirichlet Eta functions. Some examples are given for the solution of the integral (1.1), most of which are not amenable to a mathematical computer package. Finally we have developed new identities for the Euler sums $(1.2)$ in the spirit of Euler (1.3).

\section{ACKNOWLEDGEMENTS}

The author is thankful to a referee for the careful reading and considered suggestions leading to a better presented paper.

\section{REFERENCES}

[1] Borwein, D.; Borwein, J. M..; Girgensohn, R. Explicit evaluation of Euler sums. Proc. Edinburgh Math. Soc. (2) 38 (1995), no. 2, 277-294.

[2] Bailey, D. H. Borwein, J. M. Computation and structure of character polylogarithms with applications to character Mordell-TornheimWitten sums. Math. Comp. 85 (2016), no. 297, 295-324.

[3] Choi, Junesang Log-sine and log-cosine integrals. Honam Math. J. 35 (2013), no. 2, 137-146.

[4] Devoto, A. Duke, D. W. Table of integrals and formulae for Feynman diagram calculations. Riv. Nuovo Cimento (3) 7 (1984), no. 6, 1-39.

[5] Euler, L. Meditationes circa singulare serierum genus, Novi Comm. Acad. Sci. Petropol. 20 (1776), 140-186; reprinted in Opera Omnia, Ser. I, Vol. 15, B. G.Teubner, Berlin, 1927, pp. 217-267. 
[6] Flajolet, P. Salvy, B. Euler sums and contour integral representations. Experiment. Math. 7 (1998), no. 1, 15-35.

[7] Freitas, P. Integrals of polylogarithmic functions, recurrence relations, and associated Euler sums. Math. Comp. 74 (2005), no. 251, 14251440.

[8] Hoffman, M. E., Multiple harmonic series, Pacific J. Math. 152 (1992), 275-290.

[9] Kölbig, K. S. Nielsen's generalized polylogarithms. SIAM J. Math. Anal. 17 (1986), no. 5, 1232-1258.

[10] Lewin, R. Polylogarithms and Associated Functions. North Holland, New York, 1981.

[11] Markett, C. Triple sums and the Riemann zeta function. J. Number Theory 48 (1994), no. 2, 113-132.

[12] Sitaramachandra Rao, R. A formula of S. Ramanujan. J. Number Theory 25 (1987), no. 1, 1-19.

[13] Sofo, A. Polylogarithmic connections with Euler sums. Sarajevo J. Math. 12(24) (2016), no. 1, 17-32.

[14] Sofo, A. Integrals of logarithmic and hypergeometric functions. Commun. Math. 24 (2016), no. 1, 7-22.

[15] Sofo, A. and Cvijović, D. Extensions of Euler Harmonic Sums, Appl. Anal. Discrete Math. 6 (2012), 317-328.

[16] Sofo, A. Integrals of inverse trigonometric and polylogarithmic functions. Submitted, 2019.

[17] Sofo, A.; Srivastava, H. M. A family of shifted harmonic sums. Ramanujan J. 37 (2015), no. 1, 89-108.

[18] Sofo, A. New classes of harmonic number identities. J. Integer Seq. 15 (2012), no. 7, Article 12.7.4, 12 pp.

[19] Sofo, A. Families of Integrals of Polylogarithmic Functions, Special Functions and Applications. Editors Choi, J. and Shilin, I. Mathematics (2019), 7, 143; doi:10.3390/math7020143, Published by MDPI AG, Basel, Switzerland.

[20] Xu, Ce. Yan, Yuhuan. Shi, Zhijuan. Euler sums and integrals of polylogarithm functions. J. Number Theory 165 (2016), 84-108.

[21] Zagier, D. Values of zeta functions and their applications, in First European Congress of Mathematicians, Vol II (Paris, 1992), Birkhauser, Boston, 1994, pp. 497-512. 